\title{
Dinutuximab combination therapy becomes first approval for high-risk neuroblastoma
}

T The approval of dinutuximab by the US Food and Drug Administration marks the third approval for a pediatric cancer and the first for patients with high-risk neuroblastoma. ${ }^{1}$ Dinutuximab is an immunotherapeutic agent; a monoclonal antibody $(\mathrm{mAb})$ targeting a glycolipid that is highly expressed on the surface of neuroblastoma cells.

The $\mathrm{mAb}$ was approved in combination with the cytokines granulocyte macrophage colony-stimulating factor (GM-CSF) and interleukin-2 (IL-2), and the oral retinoid isotretinoin (RA). The approval was based on a pivotal, phase 3, multicenter, open-label, randomized trial conducted by the Children's Oncology Group between October 2001 and January 2009 that was stopped early after the combination demonstrated superiority over standard therapy with respect to event-free survival (EFS). ${ }^{2}$

Two hundred and twenty-six patients (mostly pediatric patients, though age up to 31 years at diagnosis was allowed) with high-risk neuroblastoma were enrolled based on the following criteria: age of $\leq 31$ years at diagnosis; completion of induction therapy, autologous stem-cell transplant (SCT), and radiation therapy, with autologous SCT performed within 9 months of initiation of induction therapy; achievement of at least partial response prior to autologous SCT; enrollment between 50-100 days after final autologous SCT; absence of progressive disease; adequate organ function; life expectancy of at least 2 months; and prior enrollment in the COG biology study (ANBL00B1). An additional 25 patients with biopsy-proven residual disease after autologous SCT were also enrolled, but were nonrandomly assigned to the immunotherapy arm and were excluded from the primary outcome analysis. Patients with systemic infections or a requirement for concomitant systemic corticosteroids or immunosuppressant usage were ineligible.

Patients were randomized 1:1 to receive either standard $\mathrm{RA}$ or dinutuximab combination therapy. Those who were randomized to the immunotherapy arm received up to 5 cycles of dinutuximab at a dose of $25 \mathrm{mg} / \mathrm{m}^{2}$ body surface area [BSA] a day administered over 4 consecutive days, in addition to concurrent daily GM-CSF in cycles 1,3 , and 5 at a dose of $250 \mu \mathrm{g} / \mathrm{m}^{2}$ a day for 14 days, starting 3 days before dinutuximab, and concurrent IL-2 in cycles 2 and

\section{What's new, what's important}

The US Food and Drug Administration (FDA) approved dinutuximab as part of first-line therapy for pediatric patients with highrisk neuroblastoma.

Dinutuximab is a GD2-binding monoclonal antibody indicated, in combination with granulocyte-macrophage colonystimulating factor, interleukin-2, and 13-cis-retinoic acid for the treatment of pediatric patients with high-risk neuroblastoma who achieve at least a partial response to prior first-line multiagent, multimodality therapy.

The pivotal study showed that after 3 years of follow-up, $63 \%$ of patients who received the dinutuximab combination were alive and free of cancer growth or recurrence, compared with $46 \%$ of patients in the control arm. In an updated analysis of survival, $73 \%$ of patients who received the dinutuximab combination were alive, compared with $58 \%$ of those in the control arm.

The FDA-approved dose is $17.5 \mathrm{mg} / \mathrm{m} 2$ administered as an IV infusion over 10-20 hours for 4 consecutive days for a maximum of 5 cycles.

Dinutuximab carries a boxed warning that it causes severe painful neuropathy, facial and upper airway edema, dyspnea, bronchospasm, stridor, urticaria, and hypotension during or shortly after completion of the infusion. The most common side effects of dinutuximab are severe pain, fever, thrombocytopenia, infusion reactions, low blood pressure, hyponatremia, elevated liver enzymes, anemia, vomiting, diarrhea, low potassium levels in the blood, capillary leak syndrome, neutropenia and lymphopenia.

Jame Abraham, MD, FACP (abrahaj5@ccf.org)

4 administered as a continuous infusion for 4 days during week 1 at a dose of $3 \times 10^{6} \mathrm{IU} / \mathrm{m}^{2}$ a day and for 4 days in week 2 at $4.5 \times 10^{6} \mathrm{IU} / \mathrm{m}^{2}$ a day. In both treatment arms, $\mathrm{RA}$ was administered at a dose of $160 \mathrm{mg} / \mathrm{m}^{2} \mathrm{BSA} /$ day, divided into 2 doses for 14 consecutive days within each of 6 consecutive 28 -day cycles. Randomization was stratified on the basis of factors thought to potentially affect postautologous SCT outcome.

EFS, defined as the time from study enrollment until first relapse, progressive disease, secondary cancer, death,

Report prepared by Jane de Lartigue, PhD. JCSO 2015;13:344-346. @2015 Frontline Medical Communications. DOI 10.12788/jcso.0172. 


\section{Mechanism of action - dinutuximab Immunotherapy targeting neuroblastoma cells}

Cancer immunotherapy, broadly defined as drugs aimed at promoting an antitumor immune response, was named the top scientific breakthrough for 2013 by Science magazine and the American Association for the Advancement of Science, and it continues to make great strides in advancing the treatment of numerous cancer types.

In general, immunotherapy can be classified either as "active," in which the patient's immune system is actively induced to recognize tumor cells, or "passive," in which the need for an immune response is bypassed by directly administering components of the immune system to the patient. The latter components include monoclonal antibodies (mAbs) designed to specifically kill cancer cells by targeting tumor-associated antigens on their surface and exemplified by groundbreaking drugs such as trastuzumab.

Dinutuximab is a chimeric mAb (containing both mouse and human components) that targets glycolipid disialoganglioside.
GD2 is highly expressed on the surface of neuroblastoma cells, with limited expression in normal tissues, so it makes an ideal therapeutic target for this rare pediatric cancer.

Dinutuximab binds to GD2 on the surface of neuroblastoma cells and induces antibodydependent cellular cytotoxicity (ADCC - the antibody recruits immune effector cells via its fragment crystallizable [Fc] region) and complementdependent cytotoxicity (the antibody induces the complement system, a cascade of proteins that assemble a membrane attack complex, which inserts itself into the cell membrane of the target cell), both leading to lysis of the neuroblastoma cell.

Preclinical studies suggested that combining dinutuximab with the cytokines granulocyte macrophage colony-stimulating factor and interleukin-2 could boost the ADCC activity of this $\mathrm{mAb}$, which served as the rationale for analyzing this particular combination. or last contact with patient, was not reached at a median of 3.4 years in the dinutuximab arm, compared with 1.9 years in the RA arm (hazard ratio [HR], 0.57; $P=.01$ ). Based on an additional 3 years of follow-up after the seventh interim analysis of EFS, median OS, defined as the time from study enrollment until death or last contact with patient was not yet reached in either the dinutuximab or RA arms, but there was a trend toward improved OS in the immunotherapy group. The 2-year estimates for EFS and OS were $66 \%$ and $86 \%$, respectively, in the dinutuximab arm, compared with $46 \%$ and $75 \%$ in the RA arm $(P=.01$ and .02 , respectively).

Most of the patients (80\%) were aged $\geq 1$ year and had International Neuroblastoma Staging System stage 4 disease at the time of diagnosis. Within this subgroup of patients, EFS was $63 \%$ vs $42 \%$ in the dinutuximab and RA groups, respectively, and OS was $84 \%$ vs $76 \%$. Among the 25 patients who were nonrandomly assigned to immunotherapy, the 2-year estimates were 36\% for EFS and 76\% for OS over a median follow-up of 3.6 years.

Stage 4 disease was predictive of lower EFS and diploidy (normal tumor cell DNA index) was predictive of worse OS. Meanwhile, complete or very good partial response prior to autologous SCT was predictive of both improved EFS and OS. No other factors among the 9 assessed were significantly predictive of outcome.

The safety of dinutuximab in patients with high-risk neuroblastoma has been evaluated in 1,021 patients, including both the pivotal trial and 2 single-arm clinical trials. There was an increased incidence of treatment-related adverse events (AEs) in the dinutuximab arm, though the toxic effects were mostly expected and attributable to the binding of the antibody to GD2 on normal nerve cells or to cytokine activity. Most commonly, patients treated with dinutuximab experienced pain, pyrexia, thrombocytopenia, lymphopenia, infusion reactions, hypotension, capillary leak syndrome, and hypersensitivity reactions. The most common serious AEs were infections, infusion reactions, hypokalemia, hypotension, pain, fever and capillary leak syndrome. In the pivotal trial, grade $3 / 4$ pain was experienced by $51 \%$ of patients and was reported as a serious $\mathrm{AE}$ in $6 \%$, most commonly in the abdomen and during cycle 1. Capillary leak syndrome occurred in $23 \%$ of patients, most frequently during cycles 2 and 4 involving IL-2. Grade 3/4 hypersensitivity reactions affected a quarter of patients and were also more frequent in cycles involving IL-2. 
Per the prescribing information, the approved dose of dinutuximab is $17.5 \mathrm{mg} / \mathrm{m}^{2}$ a day as a diluted intravenous infusion over 10-20 hours for 4 consecutive days for up to 5 cycles. $^{3}$ The initial infusion rate should be $0.875 \mathrm{mg} / \mathrm{m}^{2}$ an hour, gradually increasing as tolerated to a maximum of $1.75 \mathrm{mg} / \mathrm{m}^{2}$ an hour. Patients should receive pretreatment with intravenous hydration, analgesics, antihistamines, and antipyretics prior to initiation of dinutuximab, according to the guidelines laid out in the prescribing information, to help guard against some of the toxic effects of the drug.

Dinutuximab, which is marketed as Unituxin by United Therapeutics Corporation, comes with a boxed warning against infusion reactions, including facial and upper airway edema, dyspnea, bronchospasm, stridor, urticaria, and hypotension, and neuropathy, including severe neuropathic pain, peripheral sensory neuropathy, and motor neuropathy, to alert patients and healthcare providers to these potentially severe side effects. Other warnings and precautions detail the risks of capillary leak syndrome, hypotension, neurological disorders of the eye, bone marrow suppression, electrolyte abnormalities, and atypical hemolytic uremic syndrome. The prescribing information guides dose modification in the case of some of these adverse events. Patients should also be monitored for signs and symptoms of infusion reactions and systemic infections, as well as have blood pressure and peripheral blood counts checked regularly. Pregnant women should be advised of the potential risk to the fetus and the need for effective contraception during and for 2 months after treatment. Dinutuximab has not yet been studied in patients with renal or hepatic impairment.

\section{References}

1. FDA approves first therapy for high-risk neuroblastoma [FDA news release.] http://www.fda.gov/NewsEvents/Newsroom/PressAnnouncements/ucm 437460.htm. Released March 15,0, 2015. Accessed September 14, 2015.

2. Yu AL, Gilman AL, Ozkaynak MF, et al. Anti-GD2 Antibody with GM-CSF, interleukin-2, and isotretinoin for neuroblastoma. New Eng1 J Med. 2010;363:1324-1334.

3 Unituxin (dinutuximab) injection, for intravenous use. Prescribing information. United Therapeutics Corp. https://www.unituxin.com/ downloads/full-prescribing-information.pdf. Revised March 2015. Accessed September 14, 2015. 